\title{
Potential Armamentarium to Contain COVID-19 Pandemic in Developing Countries: Be Prepared and Act Timely!
}

\author{
CS Gautam $^{1,}$ Roosy Aulakh ${ }^{2 *}$ and Priyanka Bhateja ${ }^{3}$ \\ ${ }^{1}$ Professor and Head, Department of Pharmacology, Government Medical College \\ and Hospital, Chandigarh, India \\ ${ }^{2}$ Professor, Department of Pediatrics, Government Medical College and Hospital, \\ Chandigarh, India \\ ${ }^{3}$ Senior Resident, Department of Pediatrics, Government Medical College and \\ Hospital, Chandigarh, India \\ *Corresponding Author: Roosy Aulakh, Professor, Department of Pediatrics, \\ Government Medical College and Hospital, Chandigarh, India.
}

Received: March 20, 2020

Published: March 23, 2020

(C) All rights are reserved by Roosy Aulakh., et al.

\section{Abstract}

The world today has a new challenge, the novel coronavirus (2019-nCoV) pandemic which emerged in China in early December 2019 and has brought the entire world to standstill with over 2 lac cases and nearly 9000 deaths reported worldwide. As the virus has been demonstrated to have high transmissibility with humans worldwide lacking immunity, an exponential rise in cases is expected in countries where initial cases have just emerged. Though researchers have documented efficacy of anti-viral drugs like Remdesivir/Lopinavir/Ritonavir/Ribavarin in treating cases of pneumonia caused by COVID-19 [1], these drugs are neither easily available nor is it financially feasible to use these drugs in developing countries like India.

Keywords: Novel Coronavirus (2019-nCoV); Pandemic; Anti-Viral Drugs; Armamentarium

\section{Introduction}

Vaccine development is underway, but any approved and effective COVID-19 vaccine is at least 12 - 18 months away. Based on the pharmacokinetics, pharmacodynamics and recent limited data available on their use in laboratory confirmed COVID-19 cases, we suggest the following armamentarium to be kept ready to contain the spread and decrease the mortality with novel coronavirus infection especially in developing countries.

Chloroquine: It is an anti-malarial drug which is also used in extraintestinal amoebiasis.

Mechanism of action

Chloroquine, an age old anti malarial drug, is known to be inhibitor of autophagy, a lysosome-dependent mechanism of intracellular degradation [2]. The drug is known to accumulate by a weak base mechanism in the acidic food vacuoles of intraerythrocytic trophozoites preventing hemoglobin degradation from occurring in that organelle. Chloroquine and related quinolines have also been reported to inhibit the novel heme polymerase enzyme that is also present in the trophozoite food vacuole [3].

\section{Potential role in COVID-19 infection}

Chloroquine causes elevation of endosomal $\mathrm{pH}$ and interferes with terminal glycosylation of the cellular receptor, angiotensinconverting enzyme 2 (ACE 2), cell surface binding sites for S protein of SARS-CoV. This along with the elevation of vesicular $\mathrm{pH}$ may negatively influence the virus-receptor binding and abrogate the infection by SARS CoV at clinically admissible concentrations [4]. Chloroquine phosphate has been shown to have apparent efficacy and acceptable safety against COVID-19 associated pneumonia in multicenter clinical trials conducted in China. The drug is recommended to be included in the next version of the Guidelines for the Prevention, Diagnosis and Treatment of Pneumonia Caused by COVID-19 issued by the National Health Commission of the People's Republic of China for treatment of COVID-19 infection in larger populations in the future [5]. The multicenter collaboration group of Department of Science and Technology of Guangdong Province and Health Commission of Guangdong Province has included chloroquine in the recommendations regarding the prevention and treatment of COVID-19 pneumonia [6]. Chloroquine has also been reported to act as a zinc ionophore, thereby allowing extra cellular zinc to enter inside the cell and inhibit viral RNA dependant RNA polymerase which has been suggested as potential mechanism of action on COVID-19 [7]. Chloroquine has been demonstrated to result in abnormally enlarged early endosomes (EE) vesicles [8]. A recent systematic review has concluded that there is rationale, pre-clinical evidence of effectiveness and evidence of safety from long-time clinical use for other indications to justify clinical research on chloroquine in patients with COVID-19. However, the authors advise that clinical use should either adhere to the Monitored Emergency Use of Unregistered Interventions (MEURI) framework 
or be ethically approved as a trial as stated by the World Health Organization. Undoubtedly, safety data and data from high-quality clinical trials are urgently needed [9].

\section{Suggested dosage}

The Chinese expert consensus guidelines recommend chloroquine phosphate tablet, $500 \mathrm{mg}$ twice per day for 10 days for patients diagnosed as mild, moderate and severe cases of novel coronavirus pneumonia and without contraindications to chloroquine [3]. Interim guidelines for treatment of COVID-19 patients in Belgium recommend chloroquine base $600 \mathrm{mg}(10 \mathrm{mg} / \mathrm{kg})$ at diagnosis and $300 \mathrm{mg} \mathrm{(5} \mathrm{mg/kg)} \mathrm{12h} \mathrm{later,} \mathrm{followed} \mathrm{by} 300 \mathrm{mg} \mathrm{(5} \mathrm{mg/}$ $\mathrm{kg}$ ) BD up to Day 5 or chloroquine phosphate $1000 \mathrm{mg}$ at diagnosis and $500 \mathrm{mg} 12 \mathrm{~h}$ later, followed by $300 \mathrm{mg}$ BD up to day 5 [10]. However, as chloroquine is known to have large volume of distribution, we recommend a loading dose followed by maintenance dose.

\section{Side effects}

Chloroquine is well tolerated by most people, even for longterm therapy. Side effects include nausea, vomiting, diarrhea, unpleasant metallic taste, abdominal cramps, anorexia, pruritis, deafness, tinnitus, seizures, hypotension, electrocardiographic changes (bundle-branch block, atrioventricular block), cardiomyopathy, retinopathy, pancytopenia, aplastic anemia, agranulocytosis, thrombocytopenia and neutropenia.

\section{Caution advised}

For use in elderly ( $>65$ years), renal functions need to be monitored. Pregnancy is however not considered a contraindication for administration of chloroquine.

\section{Contraindications}

Chloroquine is contraindicated in psoriasis, retinal disease, or porphyria.

\section{Prophylactic role}

As the inhibitory effects of chloroquine have been observed when the cells are treated with the drug even before exposure to the virus, prophylactic advantage of this drug may play a significant role in containment of COVID-19 [11].

Hydroxychloroquine: An anti-malarial drug which is also used in rheumatoid arthritis, systemic lupus erythematosus, Q fever and porphyria cutanea tarda.

\section{Mechanism of action}

Hydroxychloroquine inhibits stimulation of the toll-like receptor (TLR) 9 family receptors which are cellular receptors for microbial products that induce inflammatory responses through activation of the innate immune system [12]. Similar to chloroquine, hydroxychloroquine is known to increase lysosomal pH in antigenpresenting cells.

\section{Potential role in COVID-19 infection}

HCQ, similar to chloroquine is a weak base that is known to elevate the $\mathrm{pH}$ of acidic intracellular organelles, such as endosomes/ lysosomes, essential for membrane fusion [11]. HCQ has also been documented to effectively inhibit the entry step, as well as the post-entry stages of SARS-CoV-2, similar to effect of chloroquine. Both chloroquine and hydroxychloroquine have been documented to block the transport of SARS-CoV-2 from early endosomes (EEs) to endolysosomes (ELS), which appears to be a requirement to release the viral genome as in the case of SARS-CoV. Unlike chloroquine, hydroxychloroquine has also been demonstrated to increase the size and number of ELs significantly [5]. Hydroxychloroquine is also known to inhibit cytokine production and cytokine storm has been reported in SARS-CoV-2 infection, thereby suggesting its added advantage [13]

\section{Suggested dosage}

$400 \mathrm{mg}$ at suspicion/diagnosis, $400 \mathrm{mg} 12 \mathrm{~h}$ later followed by $200 \mathrm{mg}$ BD up to Day 5 as per interim guidelines for treatment of COVID-19 patients in Belgium [6].

\section{Side effects}

Common side effects include headache, vomiting, changes in vision and muscle weakness. Severe side effects may include allergic reactions and prolongation of QT interval.

\section{Cautious use}

Advised during pregnancy, concurrent use with drugs prolonging QT interval like Azithromycin and Ondansetron which may be prescribed to patients with COVID-19 infection.

\section{Contraindications}

Hydroxychloroquine is contraindicated in myasthenia gravis, porphyria, retinal pathology, epilepsy and QTc $>500 \mathrm{msec}$.

Other drugs showing potential for use in COVID-19 infection either alone or in combination with other drugs include.

Zinc: Used to treat and prevent diarrhea in infants and children and as therapeutic drug in Wilson disease. It has been reported to reduce severity and duration of common cold and also progression of age related macular degeneration.

\section{Mechanism of action}

Zinc supplementation has been documented to decrease oxidative stress biomarkers (reactive oxygen species). Zinc supplementation results in decreased generation of inflammatory cytokines by upregulating A20, a zinc transcription factor, which inhibits the activation of NF- $\kappa B$ [14]. 


\section{Potential role in COVID-19 infection}

Increasing the concentration of intracellular zinc with zinc ionophores like pyrithione has been reported to efficiently impair the replication of a variety of RNA viruses [15]. In addition, the combination of zinc and pyrithione at low concentrations inhibits the replication of SARS coronavirus (SARS-CoV) $[16,17]$. Recently chloroquine has also been documented to increase Zinc absorption and combination of the two drugs has been proposed as an effective regimen for COVID-19 infection though large scale RCTs are required to confirm this observation [7].

Azithromycin: A macrolide antibiotic which is used in community acquired pneumonia, upper respiratory tract infections, Mycobacterium avium complex (MAC) infections in HIV patients, chancroid, chronic obstructive pulmonary disease, otitis media and urethritis.

\section{Mechanism of action}

Azithromycin inhibits RNA-dependent protein synthesis by binding to the $50 \mathrm{~S}$ ribosomal subunit resulting in blockage of transpeptidation.

\section{Role in COVID-19 infection}

Anti-inflammatory properties of Azithromycin have been well known [18]. Azithromycin has been documented to suppress Th1and Th2-related chemokines inducible protein-10 (IP-10)/macrophage-derived chemokine (MDC) in human monocytic cell lines [19]. A recent French study has documented that hydroxychloroquine was effective on its own as a treatment, when combined with azithromycin it was even more effective, and by a significant margin in confirmed cases with COVID-19 infection. Thought he results are based on limited patient size of 30 patients, they seem to be promising as they confirm similar findings reported from China [20].

\section{Side effects}

Allergic reactions (e.g. angioedema, anaphylaxis, Stevens-Johnson syndrome, toxic epidermal necrolysis and drug reaction with eosinophilia and systemic symptoms (DRESS) have been reported (rare), including fatalities; cardiac conduction defects and fungal or bacterial superinfection.

\section{Cautious use}

Advised with chloroquine or hydroxychloroquine and domperidone as it may prolong QTc interval. Use in neonates and infants may result in hypertrophic pyloric stenosis.

\section{Contraindications}

Hypersensitivity to azithromycin, erythromycin or other macrolides.

\section{Social distancing and personal hygiene}

Last but definitely not the least, social distancing by avoiding mass gatherings, unnecessary travel and maintaining 6 feet distance from others along with regular hand washing with soap and water/alcohol based sanitizer, cough etiquettes and surface disinfection with $62 \%$ - $71 \%$ ethanol, $0.5 \%$ hydrogen peroxide, or $0.1 \%$ sodium hypochlorite for 1 minute [21] are minimal pre-requisite to contain the spread of COVID-19. In addition, we should attempt to maintain adequate immunity by eating nutrient-dense diet, regularly exercising, getting adequate sleep, and controlling stress. All these measures will aid in flattening the curve of number of active cases at any given time in near future so as to avoid the health services to be overwhelmed which can prove disastrous and significantly increase mortality with the disease.

Other drugs whose potential role in COVID-19 infection may be explored further: A personal viewpoint

As the COVID-19 pandemic is showing no signs of containment and vaccine development is expected to take atleast an year or so, potential role of various other drugs in treating/limiting severity of COVID-19 infection needs to be explored urgently. Nitazoxanide is one such drug with broad spectrum antiviral activity which has been repurposed for the treatment of influenza. It has no major side effects and has been reported to inhibit the replication of coronavirus [22]. Combination of vitamin B2 and UV light has been reported to effectively reduce the titer of MERS-CoV in human plasma products [23]. Other suggested drugs which might have a potential role in COVID-19 infections include Minocycline as it is known to inhibit IL-6 and age old anti influenza drug, Amantadine, which need to be tested in clinical trials in order to document their efficacy. Other drugs which remain unexplored as of now include Topiramate (carbonic anhydrase inhibitor), Moxifloxacin (antibiotic used in respiratory illness) and Acetazolamide (carbonic anhydrase inhibitor) with role in pulmonary edema caused due to mountain sickness which may be extrapolated to potential role in COVID-19 associated ARDS.

\section{Conclusion}

As the world is taking all due measures to contain the spread of COVID-19 pandemic, the developing countries would need cheaper and readily available armamentarium to tackle this healthcare crisis. The present manuscript is an attempt to update on the existing knowledge about potential drugs which might prove to be the answer to the COVID-19 pandemic either alone or in combination with other drugs. 


\section{Bibliography}

1. Lim J., et al. "Case of the Index Patient Who Caused Tertiary Transmission of COVID-19 Infection in Korea: the Application of Lopinavir/Ritonavir for the Treatment of COVID-19 Infected Pneumonia Monitored by Quantitative RT-PCR". Journal of Korean Medical Science 35.6 (2020): e79.

2. Pasquier B. "Autophagy inhibitors". Cellular and Molecular Life Sciences 73.5 (2016): 985-1001.

3. Slater AF. "Chloroquine: mechanism of drug action and resistance in Plasmodium falciparum". Pharmacology and Therapeutics 57.2-3 (1993): 203-235.

4. Vincent MJ., et al. "Chloroquine is a potent inhibitor of SARS coronavirus infection and spread". Virology Journal 2 (2005): 69.

5. Gao J., et al. "Breakthrough: Chloroquine phosphate has shown apparent efficacy in treatment of COVID-19 associated pneumonia in clinical studies". BioScience Trends 14.1 (2020): 72 73.

6. "Expert consensus on chloroquine phosphate for the treatment of novel coronavirus pneumonia (Chinese)". Zhonghua Jie He He Hu Xi Za Zhi 43.3 (2020): 185-188.

7. Xue J., et al. "Chloroquine Is a Zinc Ionophore". PLOS One 9.10 (2014): e109180.

8. Liu J., et al. "Hydroxychloroquine, a less toxic derivative of chloroquine, is effective in inhibiting SARS-CoV-2 infection in vitro". Cell Discovery 6 (2020): 16.

9. Cortegiani A., et al. "A systematic review on the efficacy and safety of chloroquine for the treatment of COVID-19". Journal of Critical Care (2020).

10. Interim clinical guidance for patients suspected of/confirmed with COVID-19 in Belgium. Version 3 (2020).

11. Savarino A., et al. "New insights into the antiviral effects of chloroquine". Lancet Infectious Diseases 6.2 (2006): 67-69.

12. Takeda K., et al. "Toll-Like Receptors". Annual Review of Immunology 21 (2003): 335-376.

13. Huang C., et al. "Clinical features of patients infected with 2019 novel coronavirus in Wuhan, China". Lancet 395.10223 (2020): 497-506.

14. Prasad AS. "Zinc is an Antioxidant and Anti-Inflammatory Agent: Its Role in Human Health". Frontiers in Nutrition 1 (2014): 14.
15. Awotiwon AA., et al. "Zinc supplementation for the treatment of measles in children". Cochrane Database of Systematic Reviews 6 (2017): CD011177.

16. Velthuis AJW., et al. " $\mathrm{Zn}(2+)$ inhibits coronavirus and arterivirus RNA polymerase activity in vitro and zinc ionophores block the replication of these viruses in cell culture". PLOS Pathogens 6.11 (2010): e1001176.

17. Zhang L and Liu Y. "Potential interventions for novel coronavirus in China: A systematic review". Journal of Medical Virology 92.5 (2020): 479-490.

18. Ikemoto K., et al. "Contribution of anti-inflammatory and antivirulence effects of azithromycin in the treatment of experimental Staphylococcus aureus keratitis". BMC Ophthalmology 20.1 (2020): 89.

19. Kuo CH., et al. "Azithromycin suppresses Th1- and Th2-related chemokines IP-10/MDC in human monocytic cell line". Journal of Microbiology, Immunology and Infection 52.6 (2019): 872879.

20. Gautret., et al. "Hydroxychloroquine and azithromycin as a treatment of COVID-19: results of an open-label non-randomized clinical trial". International Journal of Antimicrobial Agents (2020).

21. Kampf G., et al. "Persistence of coronaviruses on inanimate surfaces and their inactivation with biocidal agents". Journal of Hospital Infection 104.3 (2020): 246-251.

22. Rossignol JF. "Nitazoxanide: a first-in-class broad-spectrum antiviral agent". Antiviral Research 110 (2014): 94-103.

23. Keil SD., et al. "Inactivation of Middle East respiratory syndrome coronavirus (MERS-CoV) in plasma products using ariboflavin-based and ultraviolet light-based photochemical treatment". Transfusion 56.12 (2016): 2948-2952.

\section{Assets from publication with us}

- Prompt Acknowledgement after receiving the article

- Thorough Double blinded peer review

- Rapid Publication

- Issue of Publication Certificate

- High visibility of your Published work Website: www.actascientific.com/ Submit Article: www.actascientific.com/submission.php Email us: editor@actascientific.com Contact us: +919182824667 